\title{
A method for estimating the power of moments
}

\author{
Shuhua Chang ${ }^{1}$, Deli $\mathrm{Li}^{2}$, Yongcheng $\mathrm{Qi}^{3}$ and Andrew Rosalsky ${ }^{4^{*}}$ (D)
}

\author{
"Correspondence: \\ rosalsky@stat.ufl.edu \\ ${ }^{4}$ Department of Statistics, University \\ of Florida, Gainesville, USA \\ Full list of author information is \\ available at the end of the article
}

$$
\begin{aligned}
& \text { Abstract } \\
& \text { Let } X \text { be an observable random variable with unknown distribution function } \\
& F(x)=\mathbb{P}(X \leq X),-\infty<x<\infty \text {, and let } \\
& \qquad \theta=\sup \left\{r \geq 0: \mathbb{E}|X|^{r}<\infty\right\} .
\end{aligned}
$$

We call $\theta$ the power of moments of the random variable $X$. Let $X_{1}, X_{2}, \ldots, X_{n}$ be a random sample of size $n$ drawn from $F(\cdot)$. In this paper we propose the following simple point estimator of $\theta$ and investigate its asymptotic properties:

$$
\hat{\theta}_{n}=\frac{\log n}{\log \max _{1 \leq k \leq n}\left|X_{k}\right|}
$$

where $\log x=\ln (e \vee x),-\infty<x<\infty$. In particular, we show that

$$
\hat{\theta}_{n} \rightarrow \mathbb{P} \theta \quad \text { if and only if } \quad \lim _{x \rightarrow \infty} x^{r} \mathbb{P}(|X|>x)=\infty \quad \forall r>\theta .
$$

This means that, under very reasonable conditions on $F(\cdot), \hat{\theta}_{n}$ is actually a consistent estimator of $\theta$.

MSC: $62 \mathrm{~F} 10 ; 60 \mathrm{~F} 15 ; 62 \mathrm{~F} 12$

Keywords: Asymptotic theorems; Consistent estimator; Point estimator; Power of moments

\section{Motivation}

The motivation of the current work arises from the following problem concerning parameter estimation. Let $X$ be an observable random variable with unknown distribution function $F(x)=\mathbb{P}(X \leq x),-\infty<x<\infty$, and let

$$
\theta=\sup \left\{r \geq 0: \mathbb{E}|X|^{r}<\infty\right\}
$$

We call $\theta$ the power of moments of the random variable $X$. Clearly $\theta$ is a parameter of the distribution of the random variable $X$. Now let $X_{1}, X_{2}, \ldots, X_{n}$ be a random sample of size $n$ drawn from the random variable $X$; i.e., $X_{1}, X_{2}, \ldots, X_{n}$ are independent and identically distributed (i.i.d.) random variables whose common distribution function is $F(\cdot)$. It is natural

(c) The Author(s) 2018. This article is distributed under the terms of the Creative Commons Attribution 4.0 International License (http://creativecommons.org/licenses/by/4.0/), which permits unrestricted use, distribution, and reproduction in any medium, provided you give appropriate credit to the original author(s) and the source, provide a link to the Creative Commons license, and indicate if changes were made. 
to pose the following question: Can we estimate the parameter $\theta$ based on the random sample $X_{1}, \ldots, X_{n}$ ?

This is a serious and important problem. For example, if $\theta>2$ and if the distribution of $X$ is nondegenerate, then it is clear that $0<\operatorname{Var} X<\infty$ and so by the classical Lévy central limit theorem, the distribution of

$$
\frac{S_{n}-n \mu}{\sqrt{n}}
$$

is approximately normal (for all sufficiently large $n$ ) with mean 0 and variance $\sigma^{2}=\operatorname{Var} X=$ $\mathbb{E}(X-\mu)^{2}$ where $\mu=\mathbb{E} X$. Thus the problem that we are facing is how can we conclude with a high degree of confidence that $\theta>2$.

In this paper we propose the following point estimator of $\theta$ and will investigate its asymptotic properties:

$$
\hat{\theta}_{n}=\frac{\log n}{\log \max _{1 \leq k \leq n}\left|X_{k}\right|}
$$

Here and below $\log x=\ln (e \vee x),-\infty<x<\infty$.

Our main results will be stated in Sect. 2 and they all pertain to a sequence of i.i.d. random variables $\left\{X_{n} ; n \geq 1\right\}$ drawn from the distribution function $F(\cdot)$ of the random variable $X$. The proofs of our main results will be provided in Sect. 3 .

\section{Statement of the main results}

Throughout, $X$ is a random variable with unknown distribution $F(x)=\mathbb{P}(X \leq x),-\infty<$ $x<\infty$ and write

$$
\rho_{1}=\sup \left\{r \geq 0: \lim _{x \rightarrow \infty} x^{r} \mathbb{P}(X>x)=0\right\} \quad \text { and } \quad \rho_{2}=\sup \left\{r \geq 0: \liminf _{x \rightarrow \infty} x^{r} \mathbb{P}(X>x)=0\right\} \text {. }
$$

Clearly, just as $\theta$ as defined in Sect. 1 is a parameter of the distribution $F(\cdot)$ of the random variable $X$, so are $\rho_{1}$ and $\rho_{2}$. These parameters satisfy

$$
0 \leq \rho_{1} \leq \rho_{2} \leq \infty
$$

The main results of this paper are Theorems 2.1-2.5.

Theorem 2.1 Let $\left\{X_{n} ; n \geq 1\right\}$ be a sequence of i.i.d. random variables drawn from the distribution function $F(\cdot)$ of the random variable $X$. Then

$$
\limsup _{n \rightarrow \infty} \frac{\log \max _{1 \leq k \leq n} X_{k}}{\log n}=\frac{1}{\rho_{1}} \quad \text { a.s. }
$$

and there exists an increasing positive integer sequence $\left\{l_{n} ; n \geq 1\right\}$ (which depends on the probability distribution of $X$ when $\rho_{1}<\infty$ ) such that

$$
\lim _{n \rightarrow \infty} \frac{\log \max _{1 \leq k \leq l_{n}} X_{k}}{\log l_{n}}=\frac{1}{\rho_{1}} \quad \text { a.s. }
$$


Theorem 2.2 Let $\left\{X_{n} ; n \geq 1\right\}$ be a sequence of i.i.d.random variables drawn from the distribution function $F(\cdot)$ of the random variable $X$. Then

$$
\liminf _{n \rightarrow \infty} \frac{\log \max _{1 \leq k \leq n} X_{k}}{\log n}=\frac{1}{\rho_{2}} \quad \text { a.s. }
$$

and there exists an increasing positive integer sequence $\left\{m_{n} ; n \geq 1\right\}$ (which depends on the probability distribution of $X$ when $\rho_{2}>0$ ) such that

$$
\lim _{n \rightarrow \infty} \frac{\log \max _{1 \leq k \leq m_{n}} X_{k}}{\log m_{n}}=\frac{1}{\rho_{2}} \quad \text { a.s. }
$$

Remark 2.1 We must point out that (2.2) and (2.4) are two interesting conclusions. To see this, let $\left\{U_{n} ; n \geq 1\right\}$ be a sequence of independent random variables with

$$
\mathbb{P}\left(U_{n}=1\right)=\mathbb{P}\left(U_{n}=3\right)=\frac{1}{2 n} \quad \text { and } \quad \mathbb{P}\left(U_{n}=2\right)=1-\frac{1}{n}, \quad n \geq 1 .
$$

Since

$$
\sum_{n=1}^{\infty} \mathbb{P}\left(U_{n}=3\right)=\sum_{n=1}^{\infty} \mathbb{P}\left(U_{n}=1\right)=\sum_{n=1}^{\infty} \frac{1}{2 n}=\infty,
$$

it follows from the Borel-Cantelli lemma that

$$
\limsup _{n \rightarrow \infty} U_{n}=3 \quad \text { a.s. } \text { and } \quad \liminf _{n \rightarrow \infty} U_{n}=1 \text { a.s. }
$$

However, for any sequences $\left\{l_{n} ; n \geq 1\right\}$ and $\left\{m_{n} ; n \geq 1\right\}$ of increasing positive integers,

neither $\lim _{n \rightarrow \infty} U_{l_{n}}=3$ a.s. nor $\lim _{n \rightarrow \infty} U_{m_{n}}=1$ a.s. holds.

Remark 2.2 For an observable random variable $X$, it is often the case that $\rho_{1}=\rho_{2}$. However, for any given constants $\rho_{1}$ and $\rho_{2}$ with $0 \leq \rho_{1}<\rho_{2} \leq \infty$, one can construct a random variable $X$ such that

$$
\sup \left\{r \geq 0: \lim _{x \rightarrow \infty} x^{r} \mathbb{P}(X>x)=0\right\}=\rho_{1} \quad \text { and } \quad \sup \left\{r \geq 0: \liminf _{x \rightarrow \infty} x^{r} \mathbb{P}(X>x)=0\right\}=\rho_{2} \text {. }
$$

For example, if $0<\rho_{1}<\rho_{2}<\infty$, a random variable $X$ can be constructed having probability distribution given by

$$
\mathbb{P}\left(X=d_{n}\right)=\frac{c}{d_{n}^{\rho_{1}}}, \quad n \geq 1,
$$

where $d_{n}=2^{\left(\rho_{2} / \rho_{1}\right)^{n}}, n \geq 1$ and

$$
c=\left(\sum_{n=1}^{\infty} \frac{1}{d_{n}^{\rho_{1}}}\right)^{-1}>0
$$

Combining Theorems 2.1 and 2.2, we establish a law of large numbers for $\log \max _{1 \leq k \leq n} X_{k}, n \geq 1$ as follows. 
Theorem 2.3 Let $\left\{X_{n} ; n \geq 1\right\}$ be a sequence of i.i.d. random variables drawn from the distribution function $F(\cdot)$ of the random variable $X$ and let $\rho \in[0, \infty]$. Then the following four statements are equivalent:

$$
\begin{aligned}
& \frac{\log \max _{1 \leq k \leq n} X_{k}}{\log n} \stackrel{\text { a.s. }}{\rightarrow} \frac{1}{\rho}, \\
& \frac{\log \max _{1 \leq k \leq n} X_{k}}{\log n} \stackrel{\mathbb{P}}{\rightarrow} \frac{1}{\rho}, \\
& \rho_{1}=\rho_{2}=\rho, \\
& \lim _{x \rightarrow \infty} x^{r} \mathbb{P}(X>x)= \begin{cases}0 & \forall r<\rho \text { if } \rho>0, \\
\infty & \forall r>\rho \text { if } \rho<\infty .\end{cases}
\end{aligned}
$$

If $0 \leq \rho<\infty$, then anyone of (2.5)-(2.8) holds if and only if there exists a function $L(\cdot)$ : $(0, \infty) \rightarrow(0, \infty)$ such that

$$
\mathbb{P}(X>x) \sim \frac{L(x)}{x^{\rho}} \quad \text { as } x \rightarrow \infty \text { and } \lim _{x \rightarrow \infty} \frac{\ln L(x)}{\ln x}=0 .
$$

The following result concerns convergence in distribution for $\log \max _{1 \leq k \leq n} X_{k}, n \geq 1$.

Theorem 2.4 Let $\left\{X_{n} ; n \geq 1\right\}$ be a sequence of i.i.d. random variables drawn from the distribution function $F(\cdot)$ of the random variable X. Suppose that there exist constants $0<\rho<\infty$ and $-\infty<\tau<\infty$ and a monotone function $h(\cdot):[0, \infty) \rightarrow(0, \infty)$ with $\lim _{x \rightarrow \infty} h\left(x^{2}\right) / h(x)=1$ such that

$$
\mathbb{P}(X>x) \sim \frac{(\log x)^{\tau} h(x)}{x^{\rho}} \quad \text { as } x \rightarrow \infty .
$$

Then

$$
\begin{aligned}
& \lim _{n \rightarrow \infty} \mathbb{P}\left(\log \max _{1 \leq k \leq n} X_{k} \leq \frac{\ln n+\tau \ln \ln n+\ln h(n)-\tau \ln \rho+x}{\rho}\right) \\
& \quad=\exp \left(-e^{-x}\right) \quad \forall-\infty<x<\infty .
\end{aligned}
$$

Also, by Theorems 2.1-2.3, we have the following result for the point estimator $\hat{\theta}_{n}$.

Theorem 2.5 Let $\left\{X_{n} ; n \geq 1\right\}$ be a sequence of i.i.d. random variables drawn from the distribution function $F(\cdot)$ of the random variable $X$. Let

$$
\hat{\theta}_{n}=\frac{\log n}{\log \max _{1 \leq k \leq n}\left|X_{k}\right|}, \quad n \geq 1 .
$$

Then we have

$$
\begin{aligned}
& \liminf _{n \rightarrow \infty} \hat{\theta}_{n}=\theta=\sup \left\{r \geq 0: \mathbb{E}|X|^{r}<\infty\right\} \quad \text { a.s., } \\
& \limsup _{n \rightarrow \infty} \hat{\theta}_{n}=\sup \left\{r \geq 0: \liminf _{x \rightarrow \infty} x^{r} \mathbb{P}(|X|>x)=0\right\} \quad \text { a.s., }
\end{aligned}
$$


and the following three statements are equivalent:

$$
\begin{aligned}
& \hat{\theta}_{n} \stackrel{\text { a.s. }}{\longrightarrow} \theta, \\
& \hat{\theta}_{n} \stackrel{\mathbb{P}}{\longrightarrow} \theta, \\
& \lim _{x \rightarrow \infty} x^{r} \mathbb{P}(|X|>x)=\infty \quad \forall r>\theta \text { if } \theta<\infty .
\end{aligned}
$$

If $0 \leq \theta<\infty$, then anyone of (2.12)-(2.14) holds if and only if there exists a function $L(\cdot)$ : $(0, \infty) \rightarrow(0, \infty)$ such that

$$
\mathbb{P}(|X|>x) \sim \frac{L(x)}{x^{\theta}} \quad \text { as } x \rightarrow \infty \text { and } \lim _{x \rightarrow \infty} \frac{\ln L(x)}{\ln x}=0 .
$$

Remark 2.3 Let $\left\{X_{n} ; n \geq 1\right\}$ be a sequence of i.i.d. random variables drawn from the distribution function $F(\cdot)$ of some nonnegative random variable $X$. For each $n \geq 1$, let $X_{n, 1} \leq X_{n, 2} \leq \cdots \leq X_{n, n}$ denote the order statistics based on $X_{1}, X_{2}, \ldots, X_{n}$. To estimate the tail index of $F(\cdot)$, the well-known Hill estimator, proposed by Hill [1], is defined by

$$
\hat{\alpha}_{n}=\left(\frac{1}{k_{n}} \sum_{i=1}^{k_{n}} \ln \frac{X_{n, n-i+1}}{X_{n, n-k_{n}}}\right)^{-1},
$$

where $\left\{k_{n} ; n \geq 1\right\}$ is a sequence of positive integers satisfying

$$
1 \leq k_{n}<n \quad \text { and } \quad k_{n} \rightarrow \infty \quad \text { and } \quad k_{n} / n \rightarrow 0 \quad \text { as } n \rightarrow \infty .
$$

Mason [2, Theorem 2] showed that, for some constant $\theta \in(0, \infty)$,

$$
\hat{\alpha}_{n} \stackrel{\mathbb{P}}{\longrightarrow} \theta \quad \text { for every sequence }\left\{k_{n} ; n \geq 1\right\} \text { satisfying }(2.16)
$$

if and only if

$$
\begin{aligned}
\mathbb{P}(X>x) & \sim \frac{L(x)}{x^{\theta}} \\
\quad \text { as } x & \rightarrow \infty \text { where } L(\cdot):(0, \infty) \rightarrow(0, \infty) \text { is a slowly varying function. }
\end{aligned}
$$

Since $L(\cdot)$ defined in (2.17) is a slowly varying function,

$$
\lim _{t \rightarrow \infty} \frac{\log L(t)}{\log t}=0
$$

is always true and hence (2.15) follows from (2.17). However, the following example shows that (2.15) does not imply (2.17). Thus condtion (2.15) is weaker than (2.17).

Example 2.1 Let $\left\{X_{n} ; n \geq 1\right\}$ be a sequence of i.i.d. random variables drawn from the distribution function $F(\cdot)$ of some nonnegative random variable $X$ given by

$$
F(x)=1-\exp (-\theta[\ln (x \vee 1)]), \quad x \geq 0,
$$


where $\theta \in(0, \infty)$ is the tail index of the distribution and $[t]$ denotes the integer part of $t$. Then (2.15) holds but (2.17) is not satisfied. To see this, let

$$
L(x)=\exp (\theta(\ln x-[\ln x])), \quad x \geq e .
$$

Then

$$
\mathbb{P}(X>x)=1-F(x)=x^{-\theta} L(x), \quad x \geq e .
$$

Since, for $x \geq e, 0 \leq \ln x-[\ln x] \leq 1$, we have

$$
1 \leq L(x) \leq \exp (\theta), \quad x \geq 1
$$

and hence (2.15) holds. However, for $1<a<e$ and $x_{n}=e^{n}, n \geq 1$, we have

$$
\ln \left(a x_{n}\right)-\left[\ln \left(a x_{n}\right)\right]=(n+\ln a)-[n+\ln a]=\ln a \quad \text { and } \quad \ln \left(x_{n}\right)-\left[\ln \left(x_{n}\right)\right]=n-[n]=0 .
$$

Thus, for $\theta \in(0, \infty)$,

$$
\frac{L\left(a x_{n}\right)}{L\left(x_{n}\right)}=\frac{\exp (\theta(\ln a))}{\exp (\theta \times 0)}=a^{\theta}>1, \quad n \geq 1
$$

and hence

$$
\lim _{x \rightarrow \infty} \frac{L(a x)}{L(x)}=1 \quad \text { does not hold; }
$$

i.e., $L(\cdot)$ is not a slowly varying function. Thus $(2.17)$ is not satisfied and hence, for this example, the well-known Hill estimator cannot be used to estimate the tail index $\theta$.

\section{Proofs of the main results}

Let $\left\{A_{n} ; n \geq 1\right\}$ be a sequence of events. As usual the abbreviation $\left\{A_{n}\right.$ i.o. $\}$ stands for the case that the events $A_{n}$ occur infinitely often. That is,

$$
\left\{A_{n} \text { i.o. }\right\}=\left\{\text { events } A_{n} \text { occur infinitely often }\right\}=\bigcap_{n=1}^{\infty} \bigcup_{j=n}^{\infty} A_{j} \text {. }
$$

For events $A$ and $B$, we say $A=B$ a.s. if $\mathbb{P}(A \Delta B)=0$ where $A \Delta B=(A \backslash B) \cup(B \backslash A)$. To prove Theorem 2.1, we use the following preliminary result, which can be found in Chandra [3, Example 1.6.25(a), p. 48].

Lemma 3.1 Let $\left\{b_{n} ; n \geq 1\right\}$ be a nondecreasing sequence of positive real numbers such that

$$
\lim _{n \rightarrow \infty} b_{n}=\infty
$$

and let $\left\{V_{n} ; n \geq 1\right\}$ be a sequence of random variables. Then

$$
\left\{\max _{1 \leq k \leq n} V_{k} \geq b_{n} \text { i.o. }\right\}=\left\{V_{n} \geq b_{n} \text { i.o. }\right\} \quad \text { a.s. }
$$


Proof of Theorem 2.1 Case I: $0<\rho_{1}<\infty$. For given $\epsilon>0$, let $r(\epsilon)=\left(\frac{1}{\rho_{1}}+\epsilon\right)^{-1}$. Then

$$
0<r(\epsilon)<\rho_{1}=\sup \left\{r \geq 0: \lim _{x \rightarrow \infty} x^{r} \mathbb{P}(X>x)=0\right\}
$$

and hence

$$
\sum_{n=1}^{\infty} \mathbb{P}\left(X>n^{1 / r(\epsilon)}\right)<\infty
$$

By the Borel-Cantelli lemma, (3.1) implies that

$$
\mathbb{P}\left(X_{n}>n^{1 / r(\epsilon)} \text { i.o. }\right)=0 .
$$

By Lemma 3.1, we have

$$
\left\{\frac{\log \max _{1 \leq k \leq n} X_{k}}{\log n}>\frac{1}{\rho_{1}}+\epsilon \text { i.o. }\right\}=\left\{\max _{1 \leq k \leq n} X_{k}>n^{1 / r(\epsilon)} \text { i.o. }\right\}=\left\{X_{n}>n^{1 / r(\epsilon)} \text { i.o. }\right\} \text { a.s. }
$$

and hence

$$
\mathbb{P}\left(\frac{\log \max _{1 \leq k \leq n} X_{k}}{\log n}>\frac{1}{\rho_{1}}+\epsilon \text { i.o. }\right)=0 .
$$

Thus

$$
\limsup _{n \rightarrow \infty} \frac{\log \max _{1 \leq k \leq n} X_{k}}{\log n} \leq \frac{1}{\rho_{1}}+\epsilon \quad \text { a.s. }
$$

Letting $\epsilon \searrow 0$, we get

$$
\limsup _{n \rightarrow \infty} \frac{\log \max _{1 \leq k \leq n} X_{k}}{\log n} \leq \frac{1}{\rho_{1}} \quad \text { a.s. }
$$

By the definition of $\rho_{1}$, we have

$$
\limsup _{x \rightarrow \infty} x^{r} \mathbb{P}(X>x)=\infty \quad \forall r>\rho_{1},
$$

which is equivalent to

$$
\limsup _{x \rightarrow \infty} x \mathbb{P}\left(X>x^{\left(1 / \rho_{1}\right)-\epsilon}\right)=\infty \quad \forall \epsilon>0 .
$$

Then, inductively, we can choose positive integers $l_{n}, n \geq 1$ such that

$$
1=l_{1}<l_{2}<\cdots<l_{n}<\cdots \quad \text { and } \quad l_{n} \mathbb{P}\left(X>l_{n}^{\left(1 / \rho_{1}\right)-(1 / n)}\right) \geq 2 \ln n, \quad n \geq 1 .
$$


Note that, for any $0 \leq z \leq 1,1-z \leq e^{-z}$. Thus, for all sufficiently large $n$, we have

$$
\begin{aligned}
\mathbb{P}\left(\frac{\log \max _{1 \leq k \leq l_{n}} X_{k}}{\log l_{n}} \leq \frac{1}{\rho_{1}}-\frac{1}{n}\right) & =\mathbb{P}\left(\max _{1 \leq k \leq l_{n}} X_{k} \leq l_{n}^{\left(1 / \rho_{1}\right)-(1 / n)}\right) \\
& =\left(1-\mathbb{P}\left(X>l_{n}^{\left(1 / \rho_{1}\right)-(1 / n)}\right)\right)^{l_{n}} \\
& \leq \exp \left(-l_{n} \mathbb{P}\left(X>l_{n}^{\left(1 / \rho_{1}\right)-(1 / n)}\right)\right) \\
& \leq \exp (-2 \ln n) \\
& =n^{-2} .
\end{aligned}
$$

Since $\sum_{n=1}^{\infty} n^{-2}<\infty$, by the Borel-Cantelli lemma, we get

$$
\mathbb{P}\left(\frac{\log \max _{1 \leq k \leq l_{n}} X_{k}}{\log l_{n}} \leq \frac{1}{\rho_{1}}-\frac{1}{n} \text { i.o. }\right)=0
$$

which ensures that

$$
\liminf _{n \rightarrow \infty} \frac{\log \max _{1 \leq k \leq l_{n}} X_{k}}{\log l_{n}} \geq \frac{1}{\rho_{1}} \quad \text { a.s. }
$$

Clearly, (2.1) and (2.2) follow from (3.2) and (3.3).

Case II: $\rho_{1}=\infty$. Using the same argument used in the first half of the proof for Case I, we get

$$
\limsup _{n \rightarrow \infty} \frac{\log \max _{1 \leq k \leq n} X_{k}}{\log n} \leq \epsilon \quad \text { a.s. } \forall \epsilon>0
$$

and hence

$$
\limsup _{n \rightarrow \infty} \frac{\log \max _{1 \leq k \leq n} X_{k}}{\log n} \leq 0 \quad \text { a.s. }
$$

Note that

$$
0 \leq \frac{\log \max _{1 \leq k \leq n} X_{k}}{\log n} \quad \forall n \geq 1 .
$$

We thus have

$$
\liminf _{n \rightarrow \infty} \frac{\log \max _{1 \leq k \leq n} X_{k}}{\log n} \geq 0 \quad \text { a.s. }
$$

It thus follows from (3.4) and (3.5) that

$$
\lim _{n \rightarrow \infty} \frac{\log \max _{1 \leq k \leq n} X_{k}}{\log n}=0 \quad \text { a.s. }
$$

proving (2.1) and (2.2) (with $l_{n}=n, n \geq 1$ ).

Case III: $\rho_{1}=0$. By the definition of $\rho_{1}$, we have

$$
\limsup _{x \rightarrow \infty} x^{r} \mathbb{P}(X>x)=\infty \quad \forall r>0,
$$


which is equivalent to

$$
\limsup _{x \rightarrow \infty} x \mathbb{P}\left(X>x^{r}\right)=\infty \quad \forall r>0 .
$$

Then, inductively, we can choose positive integers $l_{n}, n \geq 1$ such that

$$
1=l_{1}<l_{2}<\cdots<l_{n}<\cdots \quad \text { and } \quad l_{n} \mathbb{P}\left(X>l_{n}^{n}\right) \geq 2 \ln n, \quad n \geq 1 .
$$

Thus, for all sufficiently large $n$, we have by the same argument as in Case I

$$
\mathbb{P}\left(\frac{\log \max _{1 \leq k \leq l_{n}} X_{k}}{\log l_{n}} \leq n\right) \leq n^{-2}
$$

and hence by the Borel-Cantelli lemma

$$
\mathbb{P}\left(\frac{\log \max _{1 \leq k \leq l_{n}} X_{k}}{\log l_{n}} \leq n \text { i.o. }\right)=0
$$

which ensures that

$$
\lim _{n \rightarrow \infty} \frac{\log \max _{1 \leq k \leq l_{n}} X_{k}}{\log l_{n}}=\infty \text { a.s. }
$$

Thus (2.1) and (2.2) hold. This completes the proof of Theorem 2.1.

Proof of Theorem 2.2 Case I: $0<\rho_{2}<\infty$. For given $\rho_{2}<r<\infty$, let $r_{1}=\left(r+\rho_{2}\right) / 2$ and $\tau=1-\left(r_{1} / r\right)$. Then $\rho_{2}<r_{1}<r<\infty$ and $\tau>0$. By the definition of $\rho_{2}$, we have

$$
\lim _{x \rightarrow \infty} x^{r_{1}} \mathbb{P}(X>x)=\infty
$$

and hence, for all sufficiently large $x$,

$$
\mathbb{P}(X>x) \geq x^{-r_{1}} .
$$

Thus, for all sufficiently large $n$,

$$
n \mathbb{P}\left(X>n^{1 / r}\right) \geq n\left(n^{1 / r}\right)^{-r_{1}}=n^{1-\left(r_{1} / r\right)}=n^{\tau}
$$

and hence

$$
\mathbb{P}\left(\max _{1 \leq k \leq n} X_{k} \leq n^{1 / r}\right)=\left(1-\mathbb{P}\left(X>n^{1 / r}\right)\right)^{n} \leq e^{-n \mathbb{P}\left(X>n^{1 / r}\right)} \leq e^{-n^{\tau}} .
$$

Since

$$
\sum_{n=1}^{\infty} e^{-n^{\tau}}<\infty,
$$

by the Borel-Cantelli lemma, we have

$$
\mathbb{P}\left(\max _{1 \leq k \leq n} X_{k} \leq n^{1 / r} \text { i.o. }\right)=0
$$


which implies that

$$
\liminf _{n \rightarrow \infty} \frac{\log \max _{1 \leq k \leq n} X_{k}}{\log n} \geq 1 / r \text { a.s. }
$$

Letting $r \searrow \rho_{2}$, we get

$$
\liminf _{n \rightarrow \infty} \frac{\log \max _{1 \leq k \leq n} X_{k}}{\log n} \geq \frac{1}{\rho_{2}} \quad \text { a.s. }
$$

Again, by the definition of $\rho_{2}$, we have

$$
\liminf _{x \rightarrow \infty} x^{r} \mathbb{P}(X>x)=0 \quad \forall r<\rho_{2},
$$

which is equivalent to

$$
\liminf _{x \rightarrow \infty} x \mathbb{P}\left(X>x^{\left(1 / \rho_{2}\right)+\epsilon}\right)=0 \quad \forall \epsilon>0 .
$$

Then, inductively, we can choose positive integers $m_{n}, n \geq 1$ such that

$$
1=m_{1}<m_{2}<\cdots<m_{n}<\cdots \quad \text { and } \quad m_{n} \mathbb{P}\left(X>m_{n}^{\left(1 / \rho_{2}\right)+(1 / n)}\right) \leq n^{-2}, \quad n \geq 1 .
$$

Then we have

$$
\sum_{n=1}^{\infty} \mathbb{P}\left(\max _{1 \leq k \leq m_{n}} X_{k}>m_{n}^{\left(1 / \rho_{2}\right)+(1 / n)}\right) \leq \sum_{n=1}^{\infty} m_{n} \mathbb{P}\left(X>m_{n}^{\left(1 / \rho_{2}\right)+(1 / n)}\right) \leq \sum_{n=1}^{\infty} n^{-2}<\infty .
$$

Thus, by the Borel-Cantelli lemma, we get

$$
\mathbb{P}\left(\frac{\log \max _{1 \leq k \leq m_{n}} X_{k}}{\log m_{n}}>\frac{1}{\rho_{2}}+\frac{1}{n} \text { i.o. }\right)=0
$$

which ensures that

$$
\limsup _{n \rightarrow \infty} \frac{\log \max _{1 \leq k \leq m_{n}} X_{k}}{\log m_{n}} \leq \frac{1}{\rho_{2}} \quad \text { a.s. }
$$

Clearly, (2.3) and (2.4) follow from (3.6) and (3.7).

Case II: $\rho_{2}=\infty$. By the definition of $\rho_{2}$, we have

$$
\liminf _{x \rightarrow \infty} x^{r} \mathbb{P}(X>x)=0 \quad \forall r>0,
$$

which is equivalent to

$$
\liminf _{x \rightarrow \infty} x \mathbb{P}\left(X>x^{r}\right)=0 \quad \forall r>0 .
$$

Then, inductively, we can choose positive integers $m_{n}, n \geq 1$ such that

$$
1=m_{1}<m_{2}<\cdots<m_{n}<\cdots \quad \text { and } \quad m_{n} \mathbb{P}\left(X>m_{n}^{1 / n}\right) \leq n^{-2}, \quad n \geq 1 .
$$


Thus

$$
\sum_{n=1}^{\infty} \mathbb{P}\left(\max _{1 \leq k \leq m_{n}} X_{k}>m_{n}^{1 / n}\right) \leq \sum_{n=1}^{\infty} m_{n} \mathbb{P}\left(X>m_{n}^{1 / n}\right) \leq \sum_{n=1}^{\infty} n^{-2}<\infty
$$

and hence by the Borel-Cantelli lemma

$$
\mathbb{P}\left(\max _{1 \leq k \leq m_{n}} X_{k}>m_{n}^{1 / n} \text { i.o. }\right)=0
$$

which ensures that

$$
\limsup _{n \rightarrow \infty} \frac{\log \max _{1 \leq k \leq m_{n}} X_{k}}{\log m_{n}} \leq 0 \quad \text { a.s. }
$$

It is clear that

$$
\liminf _{n \rightarrow \infty} \frac{\log \max _{1 \leq k \leq n} X_{k}}{\log n} \geq 0 \quad \text { a.s. }
$$

It thus follows from (3.8) and (3.9) that

$$
\liminf _{n \rightarrow \infty} \frac{\log \max _{1 \leq k \leq n} X_{k}}{\log n}=0 \quad \text { a.s. } \quad \text { and } \quad \lim _{n \rightarrow \infty} \frac{\log \max _{1 \leq k \leq m_{n}} X_{k}}{\log m_{n}}=0 \text { a.s.; }
$$

i.e., (2.3) and (2.4) hold.

Case III: $\rho_{2}=0$. Using the same argument used in the first half of the proof for Case I, we get

$$
\liminf _{n \rightarrow \infty} \frac{\log \max _{1 \leq k \leq n} X_{k}}{\log n} \geq \frac{1}{r} \quad \text { a.s. } \forall r>0
$$

Letting $r \searrow 0$, we get

$$
\liminf _{n \rightarrow \infty} \frac{\log \max _{1 \leq k \leq n} X_{k}}{\log n}=\infty \text { a.s. }
$$

Thus

$$
\lim _{n \rightarrow \infty} \frac{\log \max _{1 \leq k \leq n} X_{k}}{\log n}=\infty \text { a.s. }
$$

and hence (2.3) and (2.4) hold with $m_{n}=n, n \geq 1$.

Proof of Theorem 2.3 It follows from Theorems 2.1 and 2.2 that

$$
(2.5) \Longleftrightarrow(2.7) \Longleftrightarrow(2.8) \text {. }
$$

Since (2.6) follows from (2.5), we only need to show that (2.6) implies (2.8). It follows from (2.6) that

$$
\lim _{n \rightarrow \infty} \mathbb{P}\left(\frac{\log \max _{1 \leq k \leq n} X_{k}}{\log n} \leq \frac{1}{r}\right)= \begin{cases}1 & \forall r<\rho \text { if } \rho>0 \\ 0 & \forall r>\rho \text { if } \rho<\infty\end{cases}
$$


Since, for $n \geq 3$,

$$
\mathbb{P}\left(\frac{\log \max _{1 \leq k \leq n} X_{k}}{\log n} \leq \frac{1}{r}\right)=\mathbb{P}\left(\max _{1 \leq k \leq n} X_{k} \leq n^{1 / r}\right)=\left(1-\mathbb{P}\left(X>n^{1 / r}\right)\right)^{n}=e^{n \ln \left(1-\mathbb{P}\left(X>n^{1 / r}\right)\right)}
$$

and

$$
n \ln \left(1-\mathbb{P}\left(X>n^{1 / r}\right)\right) \sim-n \mathbb{P}\left(X>n^{1 / r}\right) \quad \text { as } n \rightarrow \infty,
$$

it follows from (3.10) that

$$
\lim _{n \rightarrow \infty} n \mathbb{P}\left(X>n^{1 / r}\right)= \begin{cases}0 & \forall r<\rho \text { if } \rho>0 \\ \infty & \forall r>\rho \text { if } \rho<\infty\end{cases}
$$

which is equivalent to (2.8).

For $0 \leq \rho<\infty$, note that

$$
\mathbb{P}(X>x)=x^{-\rho}\left(x^{\rho} \mathbb{P}(X>x)\right)=e^{-\rho \ln x+\ln \left(x^{\rho} \mathbb{P}(X>x)\right)} \quad \forall x>0 .
$$

We thus see that, if $0 \leq \rho<\infty$, then (2.8) is equivalent to

$$
\lim _{x \rightarrow \infty} \frac{\ln \left(x^{\rho} \mathbb{P}(X>x)\right)}{\log x}=0 .
$$

(We leave it to the reader to work out the details of the proof.) We thus see that (2.8) implies (2.9) with $L(x)=x^{\rho} \mathbb{P}(X>x), x>0$. It is easy to verify that (2.8) follows from (2.9). This completes the proof of Theorem 2.3.

Proof of Theorem 2.4 For fixed $x \in(-\infty, \infty)$, write

$$
a_{n}(x)=\frac{\ln n+\tau \ln \ln n+\ln h(n)-\tau \ln \rho+x}{\rho} \quad \text { and } \quad b_{n}(x)=e^{a_{n}(x)}, n \geq 2 .
$$

Then

$$
b_{n}(x)=n^{1 / \rho}(\ln n)^{\tau / \rho}(h(n))^{1 / \rho} \rho^{-\tau / \rho} e^{x / \rho}, n \geq 2 .
$$

Since $h(\cdot):[0, \infty) \rightarrow(0, \infty)$ is a monotone function with $\lim _{x \rightarrow \infty} h\left(x^{2}\right) / h(x)=1, h(\cdot)$ is a slowly varying function such that $\lim _{x \rightarrow \infty} h\left(x^{r}\right) / h(x)=1 \forall r>0$ and hence

$$
h\left(b_{n}(x)\right) \sim h(n) \quad \text { as } n \rightarrow \infty .
$$

Clearly,

$$
\left(\ln b_{n}(x)\right)^{\tau} \sim \rho^{-\tau}(\ln n)^{\tau} \quad \text { as } n \rightarrow \infty .
$$


It thus follows from (2.10) that, as $n \rightarrow \infty$,

$$
\begin{aligned}
n \ln \left(1-\mathbb{P}\left(X>b_{n}(x)\right)\right) & \sim-n \mathbb{P}\left(X>b_{n}(x)\right) \\
& \sim-n \times \frac{\left(\ln \left(b_{n}(x)\right)\right)^{\tau} h\left(b_{n}(x)\right)}{\left(b_{n}(x)\right)^{\rho}} \\
& \sim-n \times \frac{\rho^{-\tau}(\ln n)^{\tau} h(n)}{n(\ln n)^{\tau} h(n) \rho^{-\tau} e^{x}} \\
& =-e^{-x}
\end{aligned}
$$

so that

$$
\begin{aligned}
\lim _{n \rightarrow \infty} \mathbb{P}\left(\log \max _{1 \leq k \leq n} X_{k} \leq a_{n}(x)\right) & =\lim _{n \rightarrow \infty} \mathbb{P}\left(\max _{1 \leq k \leq n} X_{k} \leq b_{n}(x)\right) \\
& =\lim _{n \rightarrow \infty}\left(1-\mathbb{P}\left(X>b_{n}(x)\right)\right)^{n} \\
& =\lim _{n \rightarrow \infty} e^{n \ln \left(1-\mathbb{P}\left(X>b_{n}(x)\right)\right)} \\
& =\exp \left(-e^{-x}\right) ;
\end{aligned}
$$

i.e., (2.11) holds.

Proof of Theorem 2.5 Since $\hat{\theta}_{n}=\frac{\log n}{\log \max _{1 \leq k \leq n}\left|X_{k}\right|}, n \geq 1$, Theorem 2.5 follows immediately from Theorems 2.1-2.3.

\section{Conclusions}

In this paper we propose the following simple point estimator of $\theta$, the power of moments of the random variable $X$, and investigate its asymptotic properties:

$$
\hat{\theta}_{n}=\frac{\log n}{\log \max _{1 \leq k \leq n}\left|X_{k}\right|} .
$$

In particular, we show that

$$
\hat{\theta}_{n} \rightarrow \mathbb{P} \theta \quad \text { if and only if } \quad \lim _{x \rightarrow \infty} x^{r} \mathbb{P}(|X|>x)=\infty \quad \forall r>\theta .
$$

This means that, under very reasonable conditions on $F(\cdot), \hat{\theta}_{n}$ is actually a consistent estimator of $\theta$. From Remark 2.3 and Example 2.1, we see that, for a nonnegative random variable $X, \hat{\theta}_{n}$ is a consistent estimator of $\theta$ whenever the well-known Hill estimator $\hat{\alpha}_{n}$ is a consistent estimator of $\theta$. However, the converse is not true.

The authors are grateful to the referee for carefully reading the manuscript and for offering helpful suggestions and constructive criticism which enabled them to improve the paper. The research of Shuhua Chang was partially supported by the National Natural Science Foundation of China (Grant \#: 91430108 and 11771322) and the research of Deli Li was partially supported by a grant from the Natural Sciences and Engineering Research Council of Canada (Grant \#: RGPIN-2014-05428). 
Authors' contributions

All authors contributed equally and significantly in writing this article. All the authors read and approved the final manuscript.

\section{Author details}

${ }^{1}$ Coordinated Innovation Center for Computable Modeling in Management Science, Tianjin University of Finance and Economics, Tianjin, China. ${ }^{2}$ Department of Mathematical Sciences, Lakehead University, Thunder Bay, Canada.

${ }^{3}$ Department of Mathematics and Statistics, University of Minnesota Duluth, Duluth, USA. ${ }^{4}$ Department of Statistics, University of Florida, Gainesville, USA.

\section{Publisher's Note}

Springer Nature remains neutral with regard to jurisdictional claims in published maps and institutional affiliations.

Received: 24 October 2017 Accepted: 22 February 2018 Published online: 06 March 2018

\section{References}

1. Hill, B.M.: A simple general approach to inference about the tail of a distribution. Ann. Stat. 3, 1163-1174 (1975)

2. Mason, D.M.: Laws of large numbers for sums of extreme values. Ann. Probab. 10, 754-764 (1982)

3. Chandra, T.K.: The Borel-Cantelli Lemma. Springer, Heidelberg (2012)

\section{Submit your manuscript to a SpringerOpen ${ }^{\circ}$ journal and benefit from:}

- Convenient online submission

- Rigorous peer review

- Open access: articles freely available online

- High visibility within the field

- Retaining the copyright to your article 